\title{
Content Based Video Retrieval Systems with Local Features: A Survey
}

\author{
Gowrisankar Kalakoti ${ }^{1}$, G. Prabhakaran ${ }^{2}$ and P. Sudhakar ${ }^{3}$ \\ ${ }^{1}$ Research Scholar, Annamalai University, Tamil Nadu, India \\ ${ }^{2 \& 3}$ Assistant Professor, Department of Computer Science \& Engineering, Annamalai University, Tamil Nadu, India \\ E-Mail: gowrisankar508@gmail.com,gpaucse@yahoo.com,kar.sudha@gmail.com
}

\begin{abstract}
With the improvement of mixed media information composes and accessible transfer speed there is immense interest of video retrieving frameworks, as clients move from content based recovery frameworks to content based retrieval frameworks. Determination of removed features assume an imperative job in substance based video retrieving paying little mind to video qualities being under thought. This work assists the up and coming analysts in the field of video retrieving with getting the thought regarding distinctive procedures and strategies accessible for the video recovery. These highlights are proposed for choosing, ordering and positioning as indicated by their potential enthusiasm to the client. Great feature determination likewise permits the time and space expenses of the recovery procedure to be lessened. This overview surveys the fascinating highlights that can be separated from video information for ordering and retrieving alongside likeness estimation techniques. We likewise recognize present research issues in territory of content based video retrieving frameworks.

Keywords: Retrieving Systems, Content Based Algorithm, Segmentation, Feature Extraction, Video Retrieval
\end{abstract}

\section{INTRODUCTION}

Content based video retrieving; ordering and recovery frameworks have pulled in scientists from much consistence. It is broadly acknowledged that fruitful answer for the issue of comprehension and ordering the recordings requires blend of data from various sources, for example, pictures, sound, content, and discourse and so on.

Retrieving has the accompanying attributes:

1. Considerably more extravagant substance than individual pictures;

2. Tremendous measure of raw information; and

3. Next to no earlier structure.

These qualities make the ordering and retrieving of video recordings very troublesome. Before, video databases have been moderately little, and ordering and recovery have been founded on catchphrases commented on physically. All the more as of late, these databases have turned out to be considerably bigger and content based video ordering and recovery is required, in light of the programmed examination of recordings with the base of human investment. Content based video retrieving has an extensive variety of uses, for example, speedy video perusing, examination of visual hardware trade, remote guidelines, computerized galleries, news video investigation, smart administration of the web recordings and video reconnaissance. A video may have a sound-related channel and in addition a visual channel. The accessible data from recordings incorporates the accompanying:

1. Video metadata, which are labeled writings inserted in recordings, more often than excluding title, outline, date, on-screen characters, maker, communication length, document estimation, video organizing, duplicate right, and so forth

2. Noise data from the sound-related channel.

3. Transcripts: Speech transcripts can be considered by discourse acknowledgment and subtitle writings can be perused utilizing optical character acknowledgment methods.

4. Visual data contained in the pictures themselves from the visual channel.

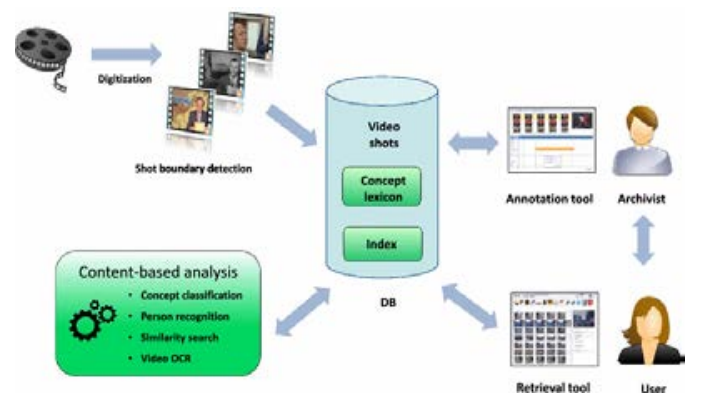

Fig. 1 Content based video Retrieval framework.

In the event that the video is incorporated into a web page, there are typically site page writings related with the video. In this paper, we center on the visual substance of recordings and give a review on visual substance based video retrieving and recovery. The significance and prevalence of video retrieving and recovery have prompted a few review papers. When all is said in done, each paper covers just a subset of the points in video ordering and recovery.

A. Video Indexing: The way toward building records for recordings regularly includes the accompanying three primary advances:

1. Video Parsing: It comprises of fleeting division of the video substance into littler units. Video parsing strategies remove basic data from the video by distinguishing transient limits and recognizing huge sections, called shots. 
2. Reflection: It comprises of extricating the agent set of video information from the video. The most generally utilized video deliberations are: the "feature" grouping and the key edge. The consequence of video deliberation frames the reason for the video ordering and perusing.

3. Content Analysis: It comprises of removing visual highlights from key edges. A few strategies utilized for picture include, extraction can be utilized be that as it may be typically stretched out to extraction of highlights that are particular to video successions, relating to the thought of protest movement, occasions and activities.

As of now the video recovery is completed in three stages:

1. Portion of a video stream into a grouping of shot, and develop a various leveled video structure dependent on shots.

2. Remove the visual element of key frames, data movement and camera parameter, at that point store them into database.

3. The framework procedure the client's inquiry and return results to the client.

A video information demonstration, which is incorporated with visual component and comment on catchphrase and a video recovery dialect on the semantic level. However, this dialect isn't helpful to the client. To encourage the client to develop an inquiry, a visual interface between the client and this dialect is as yet required at end [1]. Presently the generally acknowledged question of video is watchword and precedent inquiry.

Video division is initial move towards the substance based video seek expecting to section moving items in video arrangements. Video division at first portions the primary picture outline as the picture outline into some moving articles and after that it tracks the advancement of the moving items in the ensuing picture outlines. In the wake of portioning objects in each picture outline, these sectioned items have numerous applications, for example, observation, protest control, scene segment, and video retrieval [10]. Video is made by taking an arrangement of shots and forming them together utilizing indicated synthesis administrators. Removing structure natives is the assignment of video division that includes the recognizing of worldly limitations among scenes and between shots as appeared in Fig.2.

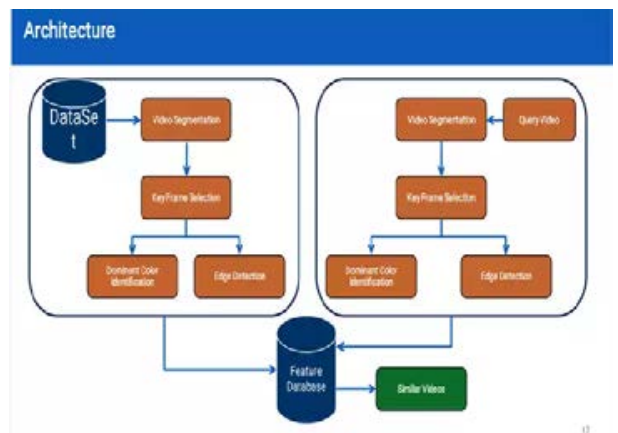

Fig. 2 Video Segmentation
The initial step for video-content examination, content based video retrieving and improvement is the dividing of a video arrangement into shots. A shot is characterized as a picture arrangement that presents nonstop activity which is caught from a solitary task of single camera. Shots are consolidated in the altering phase of video generation to shape the total arrangement. Shots can be adequately considered as the littlest ordering unit where no adjustments in scene substance can be seen and more elevated amount ideas are frequently developed by consolidating and investigating the bury and intra shot connections.

Key-outlines are still pictures separated from unique video information that best speak to the substance of shots in a theoretical way. Key-outlines have been much of the time used to enhance the content of a video log, however they were chosen physically before Key-outlines, whenever removed legitimately, are an extremely successful visual conceptual of video substance and are exceptionally valuable for quick video perusing. A video rundown, for example, a motion picture review, is an arrangement of sections from a long video program that feature the video substance, and it is most appropriate for consecutive perusing of long video programs. Aside from perusing, keyedges can likewise be utilized in speaking to video in recovery video file might be built dependent on visual highlights of key-edges, and questions might be coordinated at key-outlines utilizing inquiry by recovery calculations.

When key edges are separated following stage is to extricate highlights. The highlights are ordinarily extricated disconnected so effective calculation is certainly not a critical issue, yet huge accumulations still need a more extended time to register the highlights. Highlights of video substance can be characterized into low-level and abnormal state highlights.

\section{OVERVIEW OF CONTENT BASED VIDEO RETRIEVAL METHODS}

Video retrieval of near duplicates utilizing $\mathrm{k}-$ Nearest Neighbor (kNN) retrieval of Spatial Temporal Descriptors portrays a novel system for actualizing video look capacities, for example, recovery of close copy recordings and acknowledgment of activities in reconnaissance video. Recordings are isolated into half-second clasps whose stacked casings create 3D space-time volumes of pixels. Pixel districts with predictable shading and movement properties are removed from these 3D volumes by an edge free progressive space time division strategy. Every area is then depicted by a high-dimensional point whose segments speak to the position, introduction and, when conceivable, shade of the locale. In the ordering stage for a video database, these focuses are appointed marks that indicate their video clasp of cause.

All the marked focuses for every one of the clasps are put away into a solitary twofold tree for productive k-closest neighbor recovery. The recovery stage utilizes video 
sections as inquiries. Work introduced in fast video retrieval by means of the statistics of motion within the Regions-ofInterest (RoI) manages imperative issue to rapidly recover semantic data from a huge mixed media database. In this work, creators propose a measurement based calculation to recover the recordings that contain the protest movement from video database.

A system for video shot limits recognition and utilizing unpleasant fluffy set. They chose low-level highlights that are fundamental to accomplish high exactness for shot limit discovery. However, there are an excessive number of highlights accessible in the edge or video, for example, pixel estimations of various shading channels, measurement highlights, power and shading histogram and so on. By picking the most suitable highlights to speak to a shot or video, the computational weight will be decreased and the effectiveness will be moved forward. For this reason, the component ideal decision technique based harsh sets is presented in this area. To recognize the video shot limits, 12 hopeful highlights, characterized into 5 composes, are typically removed for basic use. The first is the RGB space display, the progressions of three hues amid shot change can be estimated, the second is HSV space show, the part of which can be estimated to the progressions of tint, immersion and incentive between nearby casings. In calculation, we register the mean of each segment of each casing in the RGB or HSV display. Due to the distinct of the target work $\mathrm{f} \mu$, one can additionally rearrange the issue by limiting $f \mu$ with regard to factors $A$ and B, separately. Distinctive strides of the calculation are given in Algorithm1.

\section{Algorithm 1}

Alternating Direction Method of Multipliers (ADMM).

Instate: A0, B0 and M0

While not united complete

Step1:A $\mathrm{t}+1=\arg \min$ A f $\mu(\mathrm{A}, \mathrm{B} \mathrm{t} ; \mathrm{M} \mathrm{t})$

Step2:B $\mathrm{t}+1=\arg \min \mathrm{B} \mathrm{f} \mu(\mathrm{At}+1, \mathrm{~B} ; \mathrm{M} \mathrm{t})$

Step3:M t+1=arg $\min \mathrm{M} \mathrm{f} \mu(\mathrm{At}+1, \mathrm{~B} \mathrm{t}+1 ; \mathrm{M})$

To start with, the competitor limits are chosen utilizing the edge based technique, and after that the SVM classifier is utilized to check the limits. The shading histogram distinction between the current edge and the past key edge to remove key casings is analyzed [2]. The author utilized the gathered vitality work processed from picture square relocations crosswise over two progressive casings to gauge the separation between edges to extricate key edges. The benefits of the successive examination based calculations incorporate their effortlessness, instinct, low computational multifaceted nature, and adjustment of the quantity of key casings to the length of the shot [1].

A two-class classifier are indentifies the isolate cuts from non cuts by SVM. A portion work is utilized to outline highlights into a high dimensional space with the end goal to conquer the impact of changes in light and quick development of items [2, 3]. In two SVM classifiers are having a sliding window, to distinguish cuts and continuous changes, individually. A few highlights from each casing, and afterward utilizes the SVM to characterize the edges utilizing these highlights into three classifications: cut, progressive change, and others $[4,5]$.

In portrays a strategy for video shot recognition utilizing low pass sifted histogram space [6]. Next the discover of shot limits among cuts and blurs/breaks down utilizing two edges and edge technique utilizing Otsu strategy to discover the edge naturally. Be that as it may, this framework was exhibited for location of cut-type shot limits [7, 8]. In model-based technique, the alter impact demonstrating progressive changes presents alter invariant property that is utilized in arranging shot limits. In which complements alter steadiness impacts by applying low pass separating to histogram contrasts between casings, while stifling movement impacts causing false alerts. Alter steadiness impacts are rectangular states of cut and triangular states of blurs/breaks up in sifted histogram contrasts in the wake of applying window convolution to unique histogram contrasts. In this manner the shot identification strategy uses low-pass channel to lessen false cautions caused by picture movement, for example, camera and items developments.

A nearest neighbor method that is the most direct way to deal with finds coordinating pictures. It contains the certain presumption that for each element the class back probabilities are roughly steady to coordinate and noncoordinating pictures. Anyway closest neighbor seeks experiences of an absence of versatility. So we have demonstrated that some well established grouping calculations can yield preferable speculation execution over closest neighbor compose calculations. Following this bearing, we utilized a characterization based PseudoRelevance Feedback (PRF) approach. The fundamental thought for our methodology is to increase the recovery execution by fusing grouping calculations by means of PRF, with the decision of preparing precedents dependent on the underlying recovery results.

A novel programmed recovery method for sight and sound information called negative pseudo-pertinence criticism [10]. It endeavors to take in a versatile closeness space via consequently nourishing back the preparation information, which is recognized dependent on a conventional likeness metric. In the undertaking of substance based video recovery, an inquiry ordinarily comprises of a content portrayal in addition to sound, pictures or video. This inquiry is presented against a video gathering. The activity of the video recovery calculation is to recover an arrangement of important video shots from a given information accumulation. In that, the positive precedents are the inquiry models and the negative models are tested from the most grounded negative models. Because of the computational issues, the input procedure rehashes for single emphasis.

The similarity measurement technique used is the accumulated difference between the current frame and the previous key frame as given in Equation 1. 


$$
N S D_{i} \quad{ }_{j}^{N} \frac{\left[H_{i}(j) H_{K F}(J)\right]^{2}}{H_{i}(j)^{2}}
$$

On the location of steady in advances, for example disintegrates and wipes which are most hard to be identified. In contrast to the unexpected cuts, the continuous progress spreads over various edges. In this strategy robotized shot limit recognition dependent on the correlation of excess of two continuous edges is utilized. Inside a worldly window we figure the common data for various sets of edges. Along these lines we make a diagram for the video arrangement where the casings are hubs and the proportions of closeness relate to the weights of the edges. By finding and detaching the frail associations between hubs we separate the chart to sub-diagrams in a perfect world comparing to the shots [11].

A two-level various leveled bunching structure to compose the substance of game recordings. The best level is grouped by shading highlight while the base level is bunched by movement inclusion. The best level contains different groups including wide-point, medium-edge and close-up shots of players from various groups. The shots inside each group are parceled to shape sub-bunches in the base level as indicated by their movement similitude. Through experimental outcomes, the author demonstrated that the cluster based retrieving, notwithstanding speed up recovery time, will by and large gives better outcomes particularly when an inquiry is situated at the limit of two groups [12].

A procedure for sectioning video is utilizing and concealed by markov demonstration [13]. It utilizes three sorts of highlights for video division, the standard histogram distinction, a sound separation measure and a gauge of protest movement between two contiguous edges. The histogram includes measures of the separation between contiguous casings dependent on the conveyance of luminance levels. The pixels are dispersed into 64 receptacles dependent on their luminance. The receptacle astute distinction of the histograms of neighboring edges is known as the histogram highlights. The sound separation is estimated by first changing over it to an arrangement of cepstal vectors, registered each $20 \mathrm{~ms}$, the probability measures are processed independently more than two contiguous interims and afterward over their connection. The proportion of the two qualities gives the probability proportion for testing the speculation that the interims speak to a similar sound compose. The movement highlight recognizes movement of articles between edges. Movement highlights are registered utilizing nine movement vectors on nine squares of the window.

\section{PROPOSED BENEATH CALCULATION MODEL}

The proposed calculation chooses the keyframes by figuring normal mean of each edge of the individual shots that are additionally put away in a vector. The neighborhood minima and maxima of every vector are then decided and are contrasted and the mean qualities, which are put away in a vector. The casings that match its mean an incentive with the nearby maxima and minima are chosen as the keyframes and their lists are noted down.

The versatile keyframe choice calculation is given in beneath calculation Algorithm 2:

To discover keyframes from the video shot Information: shots of the MPEG video

Result: kf1, kf2, kfn/Keyframes of the shots

\section{Algorithm 2:}

step 1 For every one of the shots are assigned as st1, st2, ... ,stn

step 2 For all casings $\mathrm{f} 1, \mathrm{f} 2, \ldots, \mathrm{fn}$

step 3 Register mean of each edge and store them in a vector

step 4 Process the neighborhood minima and maxima of the normal mean qualities and match them with mean qualities

step 5 Recognize the edges and check them as keyframes and note the lists

step 6 Stop

A framework utilizes syntactic model as the reason for coordinating and apply either Query-by-model or Querythrough-discourse box to interface with the client [14]. Along these lines, they work at a lower level of deliberation and in this manner; the client should be very versed in the points of interest of the CBR framework to exploit them. Prominent programmed picture ordering frameworks utilize client made inquiries, which are given through the discourse box. Anyway this technique isn't helpful as the client has to know the correct points of interest of the qualities and their usage and also subtle elements of the hunt strategy. Be that as it may, the task of such frameworks is exceedingly specialized.

A Video recovery method is still at its fundamental state, notwithstanding the way that recordings notwithstanding picture data, comprise of additional dimensional data. Three issues that have been endeavored are recover comparative recordings; find comparable video cuts in a video recover comparative shots. When all is said in done, likeness measure should be possible by coordinating highlights either locally or all inclusive. Nearby coordinating requires adjusting and coordinating edges crosswise over time [15]. For example, a unique programming is designed to adjust two video groupings of various transient lengths. Worldwide coordinating, then again, measures the comparability between two shots by figuring the separation between the two delegate highlights of shots [16].

\section{EXPERIMENTS AND CHALLENGES}

With absence of fulfillment from printed based video recovery, content based video recovery has been the consideration for specialists since long time. In the start of substance based video recovery, they endeavored to recover 
recordings utilizing a picture. Be that as it may, video recovery utilizing inquiry by picture isn't fruitful as it can't speak to a video.

A video is a succession of pictures and sound. An inquiry video gives rich substance data than that given by a question picture. Finding the important video by successively looking at the low level visual highlights of key edges of the question video with those of key edges of recordings in database give long pending answer for yield better result of video recovery. Discovering comparability measure requires key casings coordinating and subsequently figuring key edge highlights including shading histogram, surface and edge highlights, and so forth to ascertain separate parameter. These tremendous calculations cause long reaction time to the clients and in this manner, the issue of high calculation cost in figuring visual highlights of recordings is determined. Aside from this, contemplations for movement highlights, fleeting, grouping and term of shots in a video represent a test for the exploration area. The basic and substance characteristics got through substance examination, division, video parsing, reflection forms and the properties entered physically are alluded to as metadata. Video is filed on a table utilizing the metadata utilizing bunching process that arranges video clasps or shots. Bunching process orders video clasps or shots utilizing metadata to shape a list table of recordings into various visual classifications.

\section{CONCLUSION}

We have introduced a survey on late improvements in visual content based video retrieving and improvement method. The best in class of existing methodologies in each real issue has been portrayed with the attention on the accompanying errands: video structure investigation including shot limit identification, key casing extraction and scene division, highlights extraction of static key edges, questions and movements, video comment, inquiry compose and video recovery strategies, video look including interface, likeness measure and significance criticism. We have displayed an audit on ongoing improvements in content based video retrieving and enhancement methods. The cutting edge of existing methodologies in each significant issue has been depicted with the emphasis on the accompanying undertakings: video structure investigation including shot limit identification, key casing extraction and scene division, extraction of highlights of static key edges, questions and movements, video information mining, video characterization and explanation, video appended including interface, similitude measure and importance input, and video synopsis and perusing.

\section{REFERENCES}

[1] Zhenhua Guo, Lei Zhang and David Zhang, "Rotation Invariant Texture Classification using LBP Variance (LBPV) with Global Matching”, Pattern Recognition, Vol. 43, pp. 709-711, 2010.
[2] Sujuan Hou, Shangbo Zhou and Muhammad Abubakar Siddique, "Query by Example Video based on Fuzzy $C$-means Initialized by Fixed Clustering Center”, Optical Engineering, Vol. 51, No.4, pp. 047405-1, 2012.

[3] Sujuan Hou, Shangbo Zhou and Muhammad Abubakar Siddique, "A Compressed Sensing Approach for Query by Example Video Retrieval”, Multimedia Tools and Applications, Vol.72, No.3, pp. 3031-3044, 2014.

[4] David Kubon, Adam Blazek, Jakub Lokoc and Tomas Skopal, "Multi-sketch Semantic Video Browser", Multi Media Modeling, Springer, pp. 406-411, 2016.

[5] Shi Wei Lo and Fang Pang Lin, "Video Query using Temporal Signature and Similarity Matching”, Applied Mechanics and Materials, Vol. 284-287, pp. 3477-3481, 2013.

[6] Dung Mai and Kiem Hoang, "Caption Text and Keyframe based Video Retrieval System”, Computational Collective Intelligence, Technologies and Applications, Springer, pp. 244-252, 2012.

[7] B. Zhao, and E.P. Xing, "Quasi real-time summarization for consumer videos”, IEEE Conference on Computer Vision and Pattern Recognition (CVPR), IEEE, pp. 2513-2520, 2014.

[8] G. Guan, Z. Wang, S. Lu, J.D. Deng, D.D. Feng, "Keypoint-based keyframe selection", IEEE Transactions on Circuits and Systems for Video Technology, Vol.23, No.4, pp. 729-734, 2013.

[9] K. Mahmoud, N. Ghanem, and M. Ismail, "VGRAPH: An effective approach for generating static video summaries", IEEE International Conference on Computer Vision Workshops, pp. 811-818, 2013.

[10] D.J. Jeong, H.J. Yoo, and N.I. Cho, "A static video summarization method based on the sparse coding of features and representativeness of frames”, EURASIP J. Image Video Process, Vol. 123, pp.1-14, 2016.

[11] A.G. Money, and H. Agius, "Video summarisation: a conceptual framework and survey of the state of the art", J. Visual Communication and Image Representation, Vol.19, No.2, pp. 121143, 2008.

[12] G. Eason, Weining Hu, NianhuaXie, LI LI, Xianglin Zeng, and Stephan Maybank, "A survey on visual content-based video indexing and retrieval”, IEEE Transactions on systems, man and cyberneticspart c: Applications and Reviews, Vol.41, No.6, 2011.

[13] Xiaohua Duan, Liang Lin, and Hongyang Chao, "Discovering Video Shot Categories by Unsupervised Stochastic Graph Partition", IEEE Transactions on Multimedia, Vol.15, No.1, pp. 167-75, 2013.

[14] T. Yao, Y. Liu, C.-W. Ngo, and T. Mei, "Unified entity search in social media community”, In Proceedings of the 22nd International Conference on World Wide Web, ACM, pp. 1457-1466, 2013.

[15] Y. Pan, Y. Li, T. Yao, T. Mei, H. Li, and Y. Rui, "Learning deep intrinsic video representation by exploring temporal coherence and graph structure", In Proceedings of Twenty-Fifth International Joint Conference on Artificial Intelligence (IJCAI-16), pp. 3832-3838, 2016.

[16] Y. Pan, T. Yao, T. Mei, H. Li, C.-W. Ngo, and Y. Rui, " Clickthrough-based Cross-view Learning for Image Search", In Proceedings of the 37th International ACM SIGIR Conference on Research \& Development in Information Retrieval, ACM, pp. 717726, 2014.

[17] T. Yao, Y. Pan, C.W. Ngo, H. Li, and T. Mei, "Semi-supervised Domain Adaptation with Subspace Learning for Visual Recognition", IEEE Conference on Computer Vision and Pattern Recognition (CVPR), pp. 2142-2150, 2015.

[18] H. Lee, A. Battle, R. Raina, A.Y. Ng, "Efficient sparse coding algorithms", In Proceedings of International Conference on Neural Information Processing Systems, pp. 801-808, 2006.

[19] Yingwei Pan, Ting Yao, Houqiang Li, and Tao Mei, "Video Captioning with Transferred Semantic Attributes", In Proceedings of Conference on Computer Vision and Pattern Recognition, pp. 65046512, 2016.

[20] Z. Qiu, T. Yao, and T. Mei, "Deep quantization: encoding convolutional activations with deep generative model”, IEEE Conference on Computer Vision and Pattern Recognition, pp. 40854094, 2017. 